\title{
The probabilistic life table and its applications to Canada
}

\author{
$\operatorname{Nan} \operatorname{Li}^{1}$
}

\begin{abstract}
Traditionally, life-table variables are assigned certain values at all ages, and hence are treated as deterministic. But the survival process of the hypothetic cohort is uncertain; hence, the nature of life tables is probabilistic. This paper introduces a newly developed method to produce the probabilistic life table, in which each variable has a probability distribution rather than a sample value, at all ages except the first one. Applying the probabilistic life table to Canada, it concludes that the recent changes in mortality are statistically significant mostly at old ages, which cannot be estimated by the traditional life table.
\end{abstract}

Keywords: life table, probabilistic life table, survival uncertainty, significance test.

\section{Résumé}

Traditionnellement les variables des tables de mortalité sont estimées déterministiquement. Mais le processus de survie de la cohorte hypothétique est incertain; et donc les tables de mortalité sont intrinsèquement probabilistes. Cet article présente une nouvelle méthode pour produire une table de mortalité probabiliste, dans laquelle chaque variable a une distribution de probabilité plutôt qu'une valeur d'échantillon, à tous les âges, sauf au tout premier. En appliquant ce type d'approche probabiliste au Canada, on peut conclure que les récents changements de la mortalité sont statistiquement significatifs aux âges avancés, et ces progrès ne peuvent être estimés que probabilistiquement.

Mots-clés: table de mortalité, table de mortalité probabiliste, incertitude quant à la survie, test d'hypothèse.

\section{Introduction}

A life table describes the survival process of a hypothetical cohort that is closed to migration and subject to given probabilities of death by age. In the first life table, compiled by John Graunt in 1662 (see Pollard 1973), the starting number of the cohort at age 0 , namely $l_{0}$, was taken as 100 in the manner of giving examples. The current value of $l_{0}$ is commonly taken as 100,000 for no particular reason. Does the value of $l_{0}$ matter? It matters if the uncertainty of the survival process is to be explored.

In the traditional life table, besides the arbitrary starting number, the survival process from one age to another is deterministic. For example, when the probability of dying between birth and age 1 is 0.5 , the traditional life table claims that among 100 births, 50 would survive to age 1 . In reality, however, tossing 100 coins does not guarantee the occurrence of 50 heads. In other words, the survival

1. Nan Li, DC2-1938, United Nations Population Division, New York NY 10017 USA. Email: li32@un.org. 
process is uncertain, not deterministic. It should also be clarified that the "hypothetical" cohort does not mean the cohort follows an unreal survival process. To be specific, the hypothetical cohort is designed to reflect the real survival process in a hypothetical situation, where there are no effects of migration or other exiting events other than death.

The arbitrary starting number and deterministic survival process are undesirable features associated with the traditional life table. Li and Tuljapurkar $(2012,2013)$ developed a method that removes the two features and extends the life table from deterministic to probabilistic.

\section{The starting number}

The variance of the average age at death of 100 births is intuitively larger than that of 100,000 births (Li and Tuljapurkar 2012). Then what should be the value of $l_{0}$ for a specific population in a given year? If the population is stationary, the value of $l_{0}$ is obviously the number of annual births. When the observed population is not stationary, $l_{0}$ should differ from the number of annual births, according to the difference between the age structures of the observed and stationary population. For observed populations that are not stationary, Li and Tuljapurkar (2012) suggest to estimate the stationary-equivalent population, which is a hypothetic cohort with a specific starting number that minimizes the difference between the person-years of the cohort and the observed population. Let the person-years in the age interval $(a, a+n)$ be $p_{0}(a, a+n)$ and ${ }_{n} L_{a}$ for the observed and stationary population (with a starting number of 1 ), respectively. Then, the starting number of the stationaryequivalent population is ${ }^{2}$

$$
l_{0}=\frac{\sum_{a=0}^{\omega} p_{o}(a, a+n)_{n} L_{a}}{\sum_{a=0}^{\omega}{ }_{n} L_{a}^{2}},
$$

where $\omega$ indicates the lower bound of the open age group. Computing $l_{0}$ does not require data additional to that of a traditional life table. Hereafter in this paper, the value of $l_{0}$ is computed by (1), no longer 100,000 or any other arbitrary count; and the stationary-equivalent population and the hypothetical cohort will not be distinguished unless necessary.

Data on Canadian women, for the latest available years 2008 and 2009 in the Human Mortality Database (HMD, http://www.mortality.org/), are collected to demonstrate the applications of the probabilistic life table. The observed and stationary-equivalent populations of 2009 are shown in Figure 1.

Among the hypothetical cohorts of all sizes, the stationary-equivalent population is the closest to the observed population. Nonetheless, differences between the stationary-equivalent and the observed populations are expected; and they are the reason for introducing the hypothetical cohort to measure mortality.

The differences between the age patterns of the hypothetical cohort and the observed population can be viewed as the effects of historical demographic changes, which include fertility, mortality, and migration and have nothing to do with the current mortality. Subsequently, the rationale of introducing the hypothetical cohort can be understood as to eliminate the effects of historical demographic changes on the age pattern of the observed population. According to this view, the age pattern of

2. Choosing the value of $l_{0}$ to minimise $\sum_{a=0}^{\omega}\left[p_{o}(a, a+n)-l_{0} \cdot{ }_{n} L_{a}\right]^{2}$, there is $\sum_{a=}^{\infty}\left[p_{o}(a, a+n)-l_{0}{ }_{n} L_{a}\right]_{n} L_{a}=0$,
which leads to eq(1). 


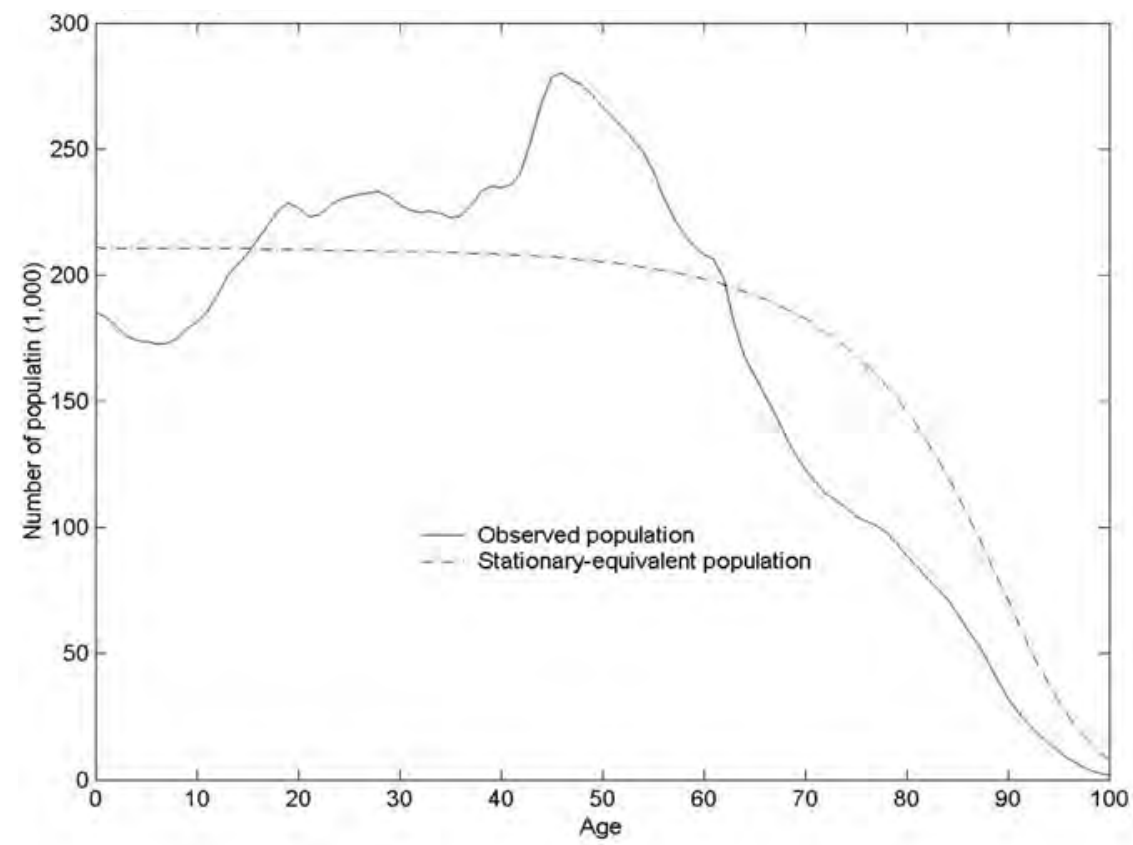

Figure 1. Age-specific numbers of Canadian women, 2009.

the hypothetical cohort would be the age pattern of the observed population, if there were no effects of historical (demographic) changes that made the age pattern of the observed population irregular.

Clearly, specifying the stationary-equivalent population is to eliminate the effects of historical events on the age-specific person-years of the observed population. As a result, the person-years of the stationary-equivalent population would be the corresponding person-years of the observed population, if there were no above-noted effects. Accordingly, the uncertain survival process of the stationary-equivalent population would be the uncertain survival process of the observed population, if again there were no above-noted effects. Subsequently, using the stationary-equivalent population, the uncertainties of the survival process of the observed population can be utilized to produce insights into the survival process that cannot be understood before. On the other hand, the survival process of a cohort with an arbitrary starting number is also uncertain, but its uncertainty is meaningless.

\section{The uncertainty}

To understand the uncertainty of the survival process, let us first define the probability of death. In statistics, the probability of dying between ages $a$ and $a+n$, namely, ${ }_{n} q_{a}$, is defined as

$$
{ }_{n} q_{a}=\frac{l_{a}-l_{a+n}}{l_{a}}
$$

when $l_{a}$ is infinitely large (see Agresti and Finlay 1997). In demography, the definition of death probability is the same as (2) but without the condition "when $l_{a}$ is infinitely large" (see Chiang 1984). Given a population in a certain year, most demographers believe that the true values of the probability of death exist; and they sometimes, if not often, need to make adjustments of the observed values to better fit the true values. But what are the true values? The true values can be defined by the statistical definition. On the other hand, the demographic definition leads to sample values of the probability of death. 
Based on the stationary-equivalent births and the statistical definition of the probability of death, Li and Tuljapurkar (2013) developed a strategy that extends the life table from deterministic to probabilistic. This new approach requires two basic assumptions. The first assumption is that the observed age-specific probabilities of death are unbiased, which differ from the true values randomly with zero mean. Accordingly, the observed values can be described as the true values plus random variables with zero mean; and these random variables describe the uncertainty. Starting from the stationary-equivalent births and using the unbiased observed probabilities of death by age, the probability distributions of the estimate true values of all life-table variables at each age can be derived.

The second assumption of producing the probabilistic life table is that individuals survive independently of each other. Under this assumption, the sample value of survivors at a certain age can be computed by the Bernoulli distribution, according to the observed probability of death and the sample value of survivors at the previous age, starting from the stationary-equivalent births. Further, the sample values of survivors at all ages compose a sample cohort; and the sample values of the probability of death by age of a sample cohort can be computed according to the demographic definition and (2). Furthermore, the sample values of all other life-table variables are calculated using the formulas in the appendix, which compose a sample life table. Finally, multiple sample life tables compose a probabilistic life table.

\section{The probabilistic life table}

In a deterministic life table, all variables depend on age only. In a probabilistic life table, all variables are random, and hence depend on age and a sample index, of which ' 0 ' is reserved for observation. More specifically, hereafter $l_{a}$ describes the number of survivors at age $a$, which is a random variable, and $l_{a}(0)$ represents the observed sample of $l_{a}$. Similarly, all life-table symbols are used to describe the corresponding random variables, and to present a sample value when an index is attached.

The first step in producing a probabilistic life table is to compute the samples of $l_{a}$ other than the observed one, namely $l_{a}(i)$, for $i=1,2,3, \ldots, S_{n}$, where $s_{n}$ stands for the number of sample life tables. The second step is to calculate the corresponding samples of other life-table variables, according to the formulas in the appendix. Using a large $S_{n}$, the sample distributions of $l_{a}$, and of all other life-table variables, are obtained.

Let ${ }_{n} q_{a}(0)$ be the unbiased observed probability of dying between age $a$ and $a+n$; then the survival process can be characterized by the random function $\Delta\left({ }_{n} q_{a}(0)\right)$ that takes value 1 to represent the survival of an individual from age $a$ to age $a+n$, or 0 to describe the death between ages $a$ and $a+n$. Under the two assumptions noted above, the survival process of an individual obeys the Bernoulli distribution, with parameter ${ }_{n} q_{a}(0)$. The values of $\Delta\left({ }_{n} q_{a}(0)\right)$ can be generated by almost any computing software. For example, if ${ }_{1} q_{0}(0)=0.5$, then among 100 values of $\Delta\left({ }_{1} q_{0}(0)\right)$, 1 would appear approximately 50 times, indicating that roughly 50 would survive to age 1 among 100 births. The exact number of $\Delta\left({ }_{1} q_{0}(0)\right)$ being 1 , however, is uncertain.

Denoting by $s(a, j)$ the survival status of the $j$ th individual at age $a$, and using $s(a, j)=1$ to represent being alive at age $a$ and $s(a, j)=0$ as being deceased before age $a$, the values of $s(a, j)$ can be computed as

$$
s(a, j)=s(a-n, j) \Delta\left({ }_{n} q_{a-n}(0)\right)
$$


For each individual, his or her $s(a, j)$ starts with 1 at age zero, and at some unpredictable age it drops to 0 , meaning death. Repeating this procedure for each of the $l_{0}$ individuals, a sample of the survival process of the cohort is obtained as below.

At any age $a$, summing up all alive individuals produces the number of survivors of the cohort, and therefore the first sample value of $l_{a}$ is obtained as:

$$
l_{a}(1)=\sum_{j=1}^{l_{0}} s(a, j)
$$

Independently regenerating another set of $\Delta\left({ }_{n} q_{a}(0)\right)$ for each of the $l_{0}$ births at all the ages, and then using (3) and (4), the values of the second sample, $l_{a}(2)$, are computed. Repeating this procedure, the sample distribution of $l_{a}$ is obtained as $l_{a}(i)$ for $i=1,2, \ldots, s_{n}$. In fact, $l_{a}(i)$ could also be computed at the cohort rather than individual level. Given the value of $l_{a-n}(i), l_{a}(i)$ could also be calculated as a random number from a binomial distribution of $l_{a-n}(i)$ trails and the probability ${ }_{n} q_{a-n}(0)$. By doing so, the computing time could be reduced significantly, but the potential of studying heterogonous models (e.g., Vaupel et al. 1979) will be lost.

Given the $i$ th sample $l_{a}(i)$, the $i$ th sample life table can be computed using the formulas in the appendix. Repeating this procedure produces $s_{n}$ sample life tables, which compose a probabilistic life table. In a probabilistic life table, the content of a variable is a probability distribution of the estimate true value of this variable, at a certain age. As a comparison, in a traditional life table, the content of a variable is only a sample of the true value.

The number of stationary-equivalent births $l_{0}$ and the number of sample cohorts $s_{n}$ are essential for the probabilistic life table. When the value of $l_{0}$ is larger, the variances of life-table variables are smaller. On the other hand, when the value of $s_{n}$ is larger, the sample distributions of these variables are smoother and more accurate.

\section{The significance test}

One important purpose of measuring mortality is to detect the difference between populations. To serve this purpose, deterministic life tables could specify whether a life-table variable for one population is bigger than this variable for another population; while a probabilistic life table can further test whether such a difference is statistically significant, or whether it may appear merely by random chance.

We denote a life-table variable for populations 1 and 2 by $x_{1}$ and $x_{2}$, and the corresponding sample values by $x_{1}(i)$ and $x_{2}(i)$, for $i=0,1,2, \ldots s_{n}$. To test the significance of the difference between the mean values of $x_{1}$ and $x_{2}$, the null hypothesis can be set as $H_{0}$ : mean $\left(x_{1}\right)=$ mean $\left(x_{2}\right)$. Under this hypothesis, the test statistic can be constructed as

$$
z=\frac{x_{1}-x_{2}-\operatorname{mean}\left(x_{1}-x_{2}\right)}{\sqrt{\hat{\sigma}_{1}^{2}+\hat{\sigma}_{2}^{2}}},
$$

where $\hat{\sigma}_{1}^{2}$ and $\hat{\sigma}_{2}^{2}$ are the estimate variances of $x_{1}$ and $x_{2}$, and can be computed from the sample distributions as

$$
\sigma_{h}^{2}=\sum_{i=1}^{s_{n}}\left[x_{h}(i)-\sum_{k=1}^{s_{n}} x_{h}(k) / s_{n}\right]^{2} /\left(s_{n}-1\right), \quad h=1,2
$$


On the other hand, without the null hypothesis, the test statistic of the observed value $z$ is

$$
z(0)=\frac{x_{1}(0)-x_{2}(0)}{\sqrt{\hat{\sigma}_{1}^{2}+\hat{\sigma}_{2}^{2}}}
$$

In the usual applications of significance test, the analytic distribution of $\mathrm{z}$ is known as standard normal, and therefore its 95 per cent confidence interval is $[-1.96,1.96]$. If $z(0)$ falls outside [-1.96,1.96], then the $H_{0}$ leads to an observation that occurs with a probability smaller than 0.05 , and hence is rejected at the 0.05 level. Subsequently, the conclusion is that the difference between the mean values of $x_{1}$ and $x_{2}$ is statistically significant. Otherwise, the difference between the means of $x_{1}$ and $x_{2}$ cannot be said statistically significant.

Here we do not know the analytic distribution, but we have the sample distribution, of $z$. Since the $i$ is chosen independently for $x_{1}(i)$ and $x_{2}(i)$, the sample distribution of $z$ can be computed as

$$
z(i)=\frac{x_{1}(i)-x_{2}(i)-\sum_{i=1}^{s_{n}}\left(x_{1}(i)-x_{2}(i)\right) / s_{n}}{\sqrt{\hat{\sigma}_{1}^{2}+\hat{\sigma}_{2}^{2}}}, \quad i=1 \sim s_{n}
$$

Using the sample distribution of $z$, we can find out the 95 per cent confidence interval, namely $\left[C_{1}, C_{2}\right]$, not exactly, but approximately. Subsequently, significance tests can be carried out following the same logic of using the standard normal distribution.

\section{Applications}

The number of sample cohorts $s_{n}$ is chosen as 1,000 in this paper. A larger $s_{n}$ will produce smoother probability distributions of all life-table variables, but on the other hand, will require more computing time. Using the 1,000 samples of life expectancy at birth, namely $e_{0}(i)$ for $i=1,2, \ldots, 1,000$ for year 2008 and 2009, two sample distributions of life expectancy at birth are obtained as can be seen in the first panel of Figure 2, in which the vertical axis indicates the number of samples in each bar. Again, the data in the application are based Canadian women in the years 2008 and 2009, the latest available in the HMD.

The first estimation of the standard errors of computing life expectancy was proposed by Chiang (1984), which inspired studies about the accuracy of computing life expectancy. These standard errors are derived from the sampling errors of the death rates of the observed populations. Because observed populations are not stationary in general, these standard errors do not measure the uncertainty of the survival process of the hypothetical cohort (Li and Tuljapurkar 2012). Assuming that the distribution of deaths within an age group is of Poisson instead of Binomial, Silcocks et al. (2001) provided another set of formulas to estimate the standard errors of life expectancy, following Chiang's method. Eayres and Willions (2004) applied the formulas of Chiang and Silcocks et al. to some hypothetical small populations with the age pattern and death rates of English men in 1998-2000. Scherbov and Ediev (2011) applied Chiang's method to stable and stationary populations with hypothetical sizes, but ignored the variance of surviving to age $a$ when estimating the standard error of deaths at ages $(a, a+n)$. These works are important and have brought due attention to the accuracy of computing life expectancy. Different from these works, the probability distributions of life expectancy at birth computed in this paper, as shown in the first panel of Figure 2, describe the uncertain survival process of the hypothetical cohort (Li and Tuljapurkar 2013). 

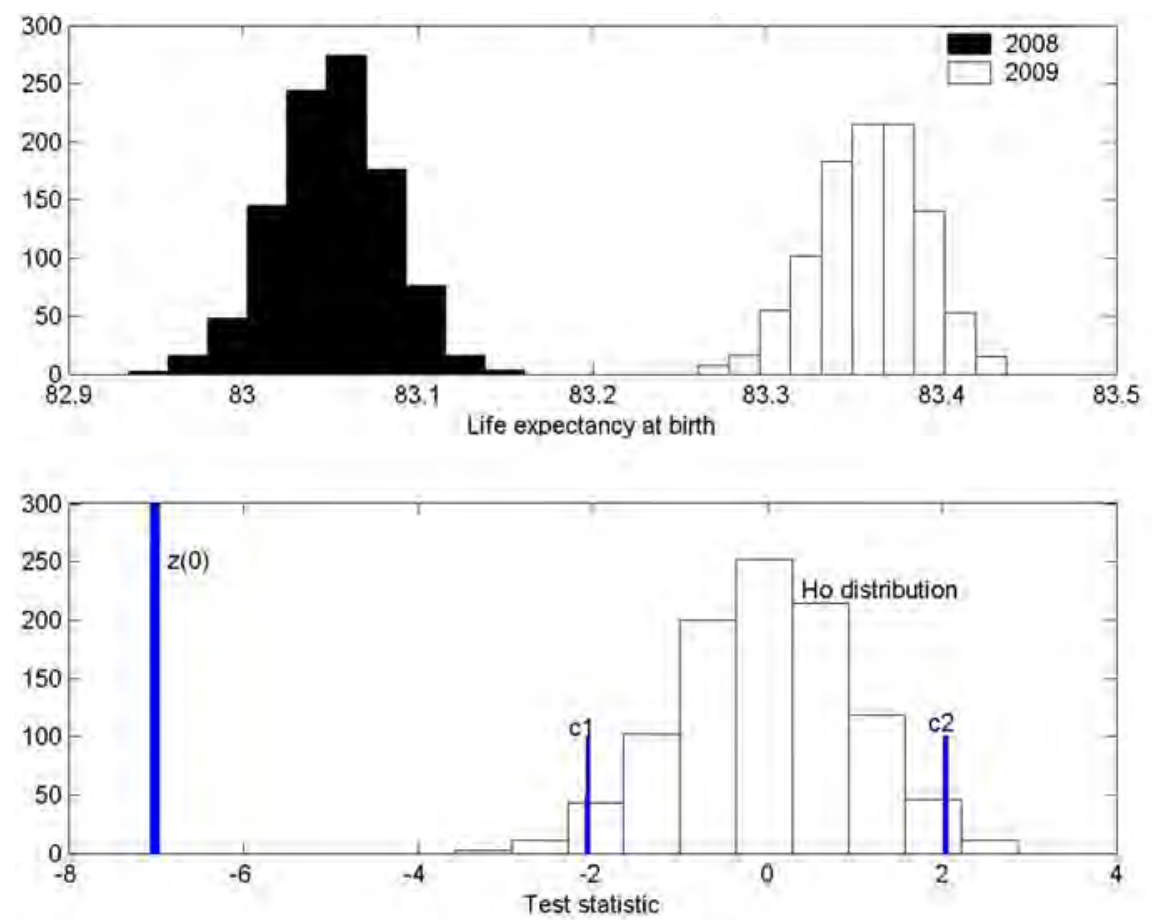

Figure 2. Distributions and significance test of life expectancy at birth, Canadian women.

Applying the above significance test to the sample distributions of life expectancy at birth of 2008 and 2009 (for Canadian women), results are shown in the second panel of Figure 2. It can be seen that the difference between the mean values of life expectancy at birth for 2008 and 2009 is significant, because $z(0)$ is outside of $\left[c_{1}, c_{2}\right]$. This conclusion is consistent with the first panel, in which the two distributions are quite distinct. The difference between life expectancies at birth is used to reflect development gaps-for example, according to the human development index of the United Nations - but whether the difference is significant or not has rarely been asked.

Infant mortality rate IMR, or $q_{0}$, which is defined as the ratio of the number of deaths at ages younger than one year to the number of births in a certain period, is perhaps the most widely estimated mortality measure. The uncertainties of ${ }_{1} q_{0}$ could be remarkable, as shown in the top panel in Figure 3 , but they are often unnoticed. Changes in infant mortality, however, can hardly be understood if these uncertainties are ignored. In fact, the ${ }_{1} q_{0}$ of Canadian females are shown as 4.68 (per thousand) in 2008 and 4.72 in 2009 in the HMD. How can there be an increase in infant mortality when the socioeconomic conditions were normal and the quality of data was high? An answer is offered from the significance test as shown in the lower panel of Figure 3: the increase was more likely just a random fluctuation, because the difference between the mean values of ${ }_{1} q_{0}$ is not statistically significant. Differences between infant mortalities are related to healthcare systems and other aspects; and again, whether the differences are significant or not had yet received adequate attention.

Adult mortality, usually defined as the probability of dying between ages 15 and 60 and denoted as ${ }_{45} q_{15}$ (per thousand), has received some research attention recently (e.g., Rajaratnam et al. 2010). The sample distributions of ${ }_{45} q_{15}$ and significance test are shown in Figure 4. It can be seen that the adult mortality of Canadian women declined between 2008 and 2009, but the decline can hardly be claimed significant, because $z(0)$ and $c_{2}$ are almost overlapped. 

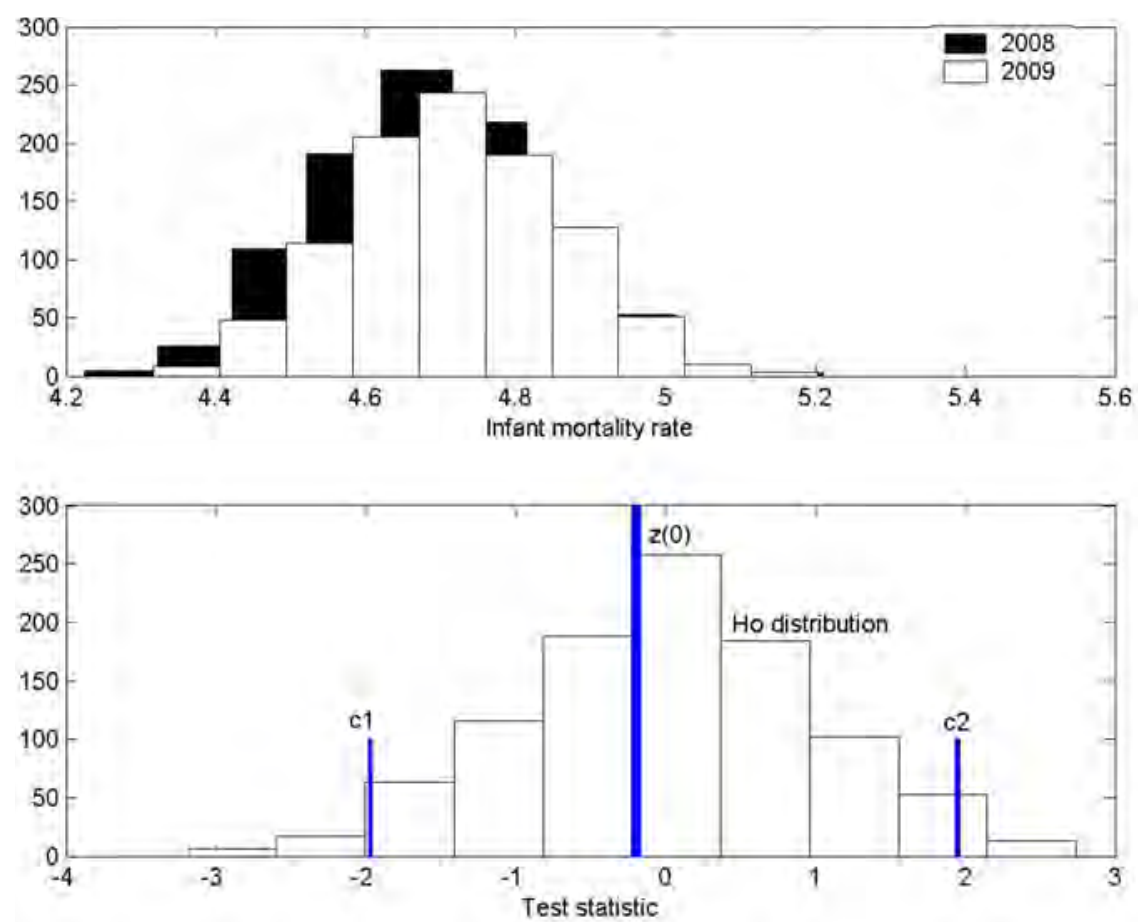

Figure 3. Distributions and significance test of infant mortality rate, Canadian women.

Comparing to infant and adult mortality, old-age mortality is perhaps more important because it covers a larger fraction of deaths among a population. In this paper, old-age mortality is measured
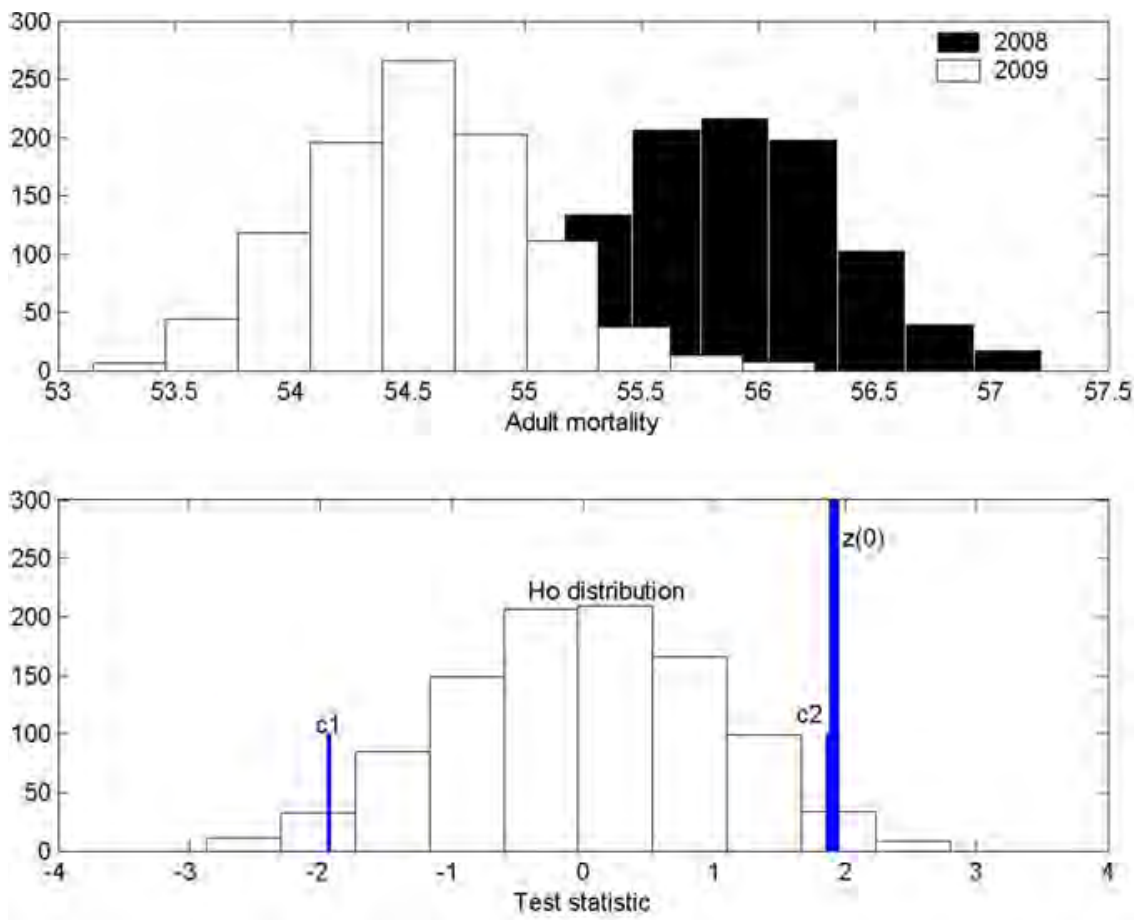

Figure 4. Distributions and significance test of adult mortality, Canadian women. 
by life expectancy at age 65 , namely $e_{65}$. The sample distributions of $e_{65}$ and significance test are displayed in Figure 5.
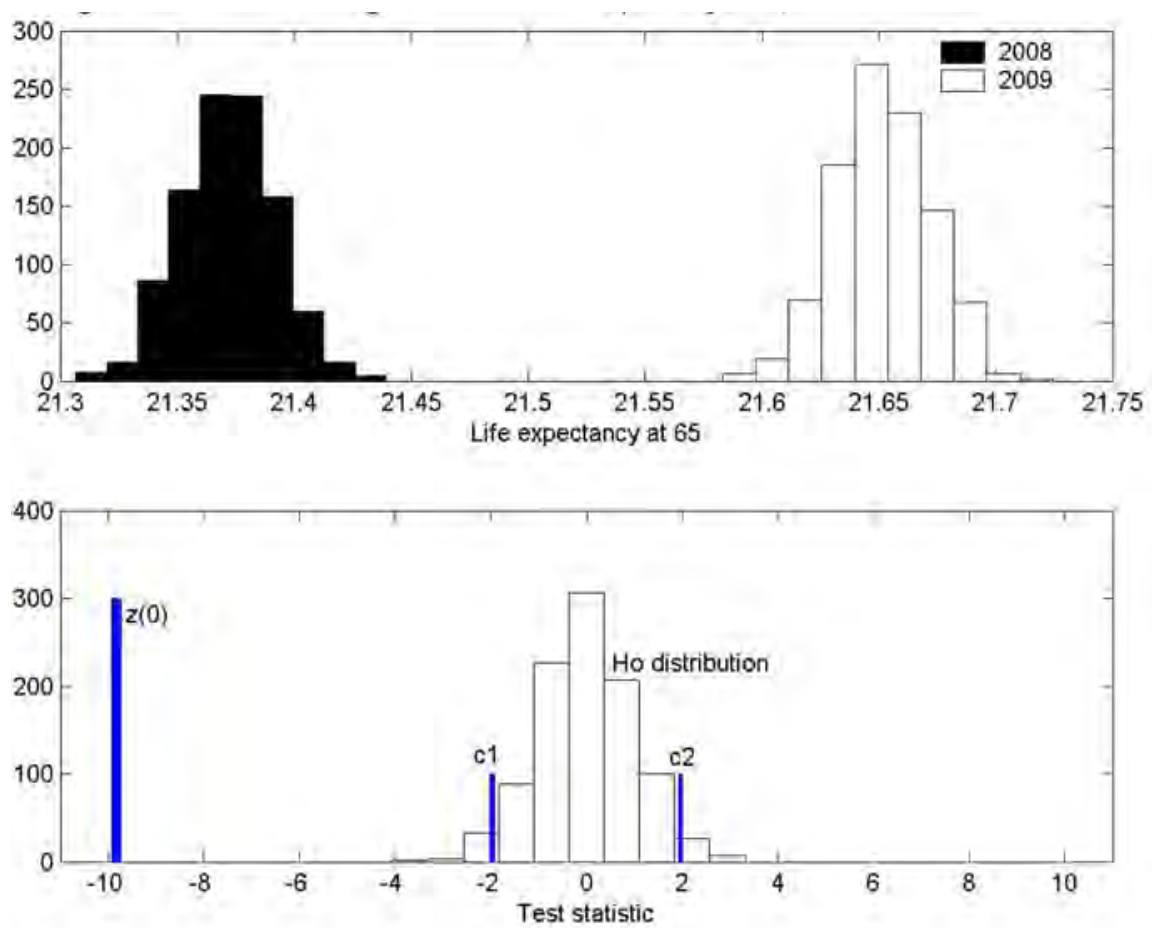

Figure 5. Distributions and significance test of life expectancy at 65, Canadian women.

It can be seen that the uncertainty of the $e_{65}$ is also remarkable, and the increase of $e_{65}$ between 2008 and 2009 is, however, statistically significant.

Finally, to illustrate the effect of population size, the uncertainties of life expectancy at birth are compared between Canada and other countries. To keep the sources of data consistent, the HMD data for 2008 and 2009 are chosen. To maximize the difference between the uncertainties, Iceland and the United States of America — with the smallest and the largest population in the HMD—are selected. In 2009, the number of women in Canada was 109 times that in Iceland, and about 11 per cent that in the USA. The probability distributions of female life expectancy in Iceland, Canada, and the USA are displayed in the three panels of Figure 6, in which the centre of each horizontal axis is the average of the corresponding mean values of life expectancy for 2008 and 2009, and the lengths of the three horizontal axes are the same, which is 2 years.

Since the lengths of the horizontal axis are the same for each country, the probability distributions are comparable. It is clear that when population size is larger, the uncertainty in estimate life expectancy is smaller. Between 2008 and 2009, the increase in the mean value of female life expectancy in Iceland was notably bigger than that in Canada and the USA. The overlap of the two distributions for Iceland in Figure 6, however, implies that the increase of female life expectancy in Iceland is not statistically significant. The significance test confirms that the bigger increase in Iceland is not statistically significant, while the smaller increases in Canada and the USA are statistically significant. These comparisons indicate that a quantitatively bigger difference may not be more likely to be significant statistically, which depends also on population size. 

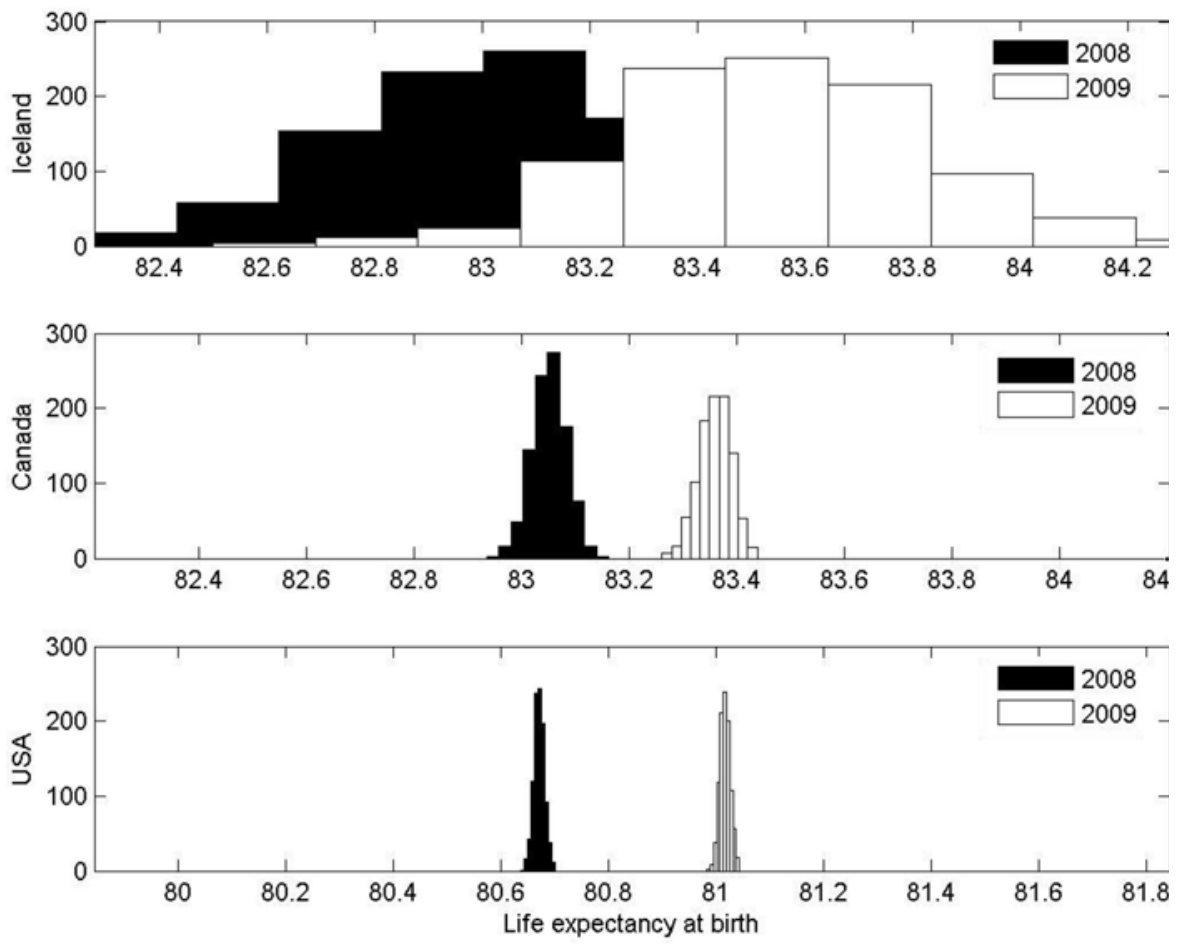

Figure 6. Distributions of female life expectancy at birth for Iceland, Canada, and the USA.

\section{Summary}

In human populations, age at death is uncertain. To make the life table consistent with this fact, $\mathrm{Li}$ and Tuljapurkar (2013) developed the probabilistic life table. This paper has introduced the probabilistic life table and applied it to Canadian women in the years 2008 and 2009.

Computing a probabilistic life table does not need data additional to that of a traditional life table, but requires two assumptions. The first assumption is that an individual survives independently from the others, and the second is that the observed age-specific probabilities of death are unbiased. The first assumption could break down when lethal and infectious diseases (such as HIV/AIDS) break out at a large scale. The second assumption implies that errors in collecting the data on death and population are negligible, so that the differences between the observed and the true values of probabilities of death are random. Clearly, these assumptions are not unreasonable in the Canadian context, given the lack of known large-scale epidemics and the high quality of Canadian vital data collected by Statistics Canada.

Applying the probabilistic life table to Canada leads to two conclusions. First, even for Canada, a country with a relatively large population, the uncertainties of life-table variables are too large to ignore. For example, Figure 2 indicates that the 95 per cent confidence intervals of life expectancy at birth contain changes in the first decimal, which implies that the customary way of using two decimals to express life expectancy can be unnecessary and misleading. Second, the annual changes of mortality are statistically significant mostly at old ages. This finding enables us to explain occasional annual increases of mortality at younger ages, which are now common in developed countries. This finding also suggests that proper attention should be paid to old-age mortality. Moreover, in unreported analyses, data on Canadian men and the year 2007 are also investigated; and the results are similar to those reported for women. 
Li: The probabilistic life table and its applications to Canada

The probabilistic life table improves mortality measures. The recent advances in probabilistic life table techniques are a result of improved computing power and increasing public interest in mortality issues. When the calculations depended on the abacus and the results were recorded on paper, the probabilistic life table was unthinkable, even if it had been developed in theory. Today, the probabilistic life table becomes a useful tool in mortality measures because calculations are now done by computers and results are stored in electronic files.

\section{Acknowledgments}

The views expressed in this paper are those of the author and do not necessarily reflect those of the United Nations. The author thanks Zheng Wu and two reviewers, as well as the editor of the journal, for their useful comments and suggestions.

\section{References}

Agresti, A., and B. Finlay. 1997. Statistical Methods for the Social Sciences. Upper Saddle River NJ: Prentice Hall.

Coale, A.J., and P. Demeny. 1966. Regional Model Life Tables and Stable Populations. Princeton NJ: Princeton University Press.

Chiang, C.L. 1984. The Life Table and Its Applications. Malabar FL: Robert E. Krieger Publishing.

Eayres, D., and E.S. Williams. 2004. Evaluation of methodologies for small area life expectancy estimation. Journal of Epidemiology and Community Health 58:243-49.

Greville, T.N.E. 1943. Short methods of constructing abridged life tables. The Record of the American Institute of Actuaries 32:29-43.

HMD. n.d. Human Mortality Database. University of California, Berkeley (USA), and Max Planck Institute for Demographic Research (Germany). Available at www.mortality.org and www.humanmortality.de (data downloaded in September 2014).

Li, N., and S. Tuljapurkar. 2013. The Probabilistic Life Table and Its Applications. Paper presented at the annual meeting of the Population Association of America, New Orleans.

- 2012. On the Accuracy of Life Expectancy. Paper presented at the annual meeting of the Population Association of America, San Francisco.

Pollard, J.H. 1973. Mathematical Models for the Growth of Human Population. Cambridge MA: Cambridge University Press.

Rajaratnam, J.K., J.R. Marcus, A. Levin-Rector, A.N. Chalupka, H. Wang, L. Dwyer, M. Costa, A.D. Lopez, and C.J.L. Murray. 2010. Worldwide mortality in men and women aged 15-59 years from 1970 to 2010: A systematic analysis. The Lancet 375:1704-20.

Scherbov, S., and D. Ediev. 2011. Significance of life table estimates for small populations: Simulationbased study of standard errors. Demographic Research 24:527-50.

Silcocks, P.B.S., D.A. Jenner, and R. Reza. 2001. Life expectancy as a summary of mortality in a population: Statistical considerations and suitability for use by health authorities. Journal of Epidemiology and Community Health 55:38-43.

Vaupel, J.W., K.G. Manton, and E. Stallard. 1979. The impact of heterogeneity in individual frailty on the dynamics of mortality. Demography 16(3):439-54. 


\section{Appendix}

In this appendix, all life table variables are deterministic; and the focus is on using $l_{a}$ to compute all other variables. We first discuss the case of abridged life tables, of which age $a$ takes the values 0 , $1,5,10$, and so on until an open age group.

Given $l_{a}$, the number of deaths ${ }_{n} d_{a}$ and the values of ${ }_{n} q_{a}$ are immediately available:

$$
\begin{aligned}
& { }_{n} d_{a}=l_{a}-l_{a+n}, \\
& { }_{n} q_{a}=\frac{{ }_{n} d_{a}}{l_{a}} .
\end{aligned}
$$

For the age group $0-1$, the calculations of death rate ${ }_{1} m_{0}$ and of person-years lived by deaths before age $1,{ }_{1} a_{0}$, are based on the results of the West Family of the Coale-Demeny (1966) model life tables:

$$
\begin{aligned}
& { }_{1} m_{0}=\left\{\begin{array}{l}
\frac{1 q_{0}}{1-\left(1-b_{0}\right) \cdot{ }_{1} q_{0}}, \text { if } \frac{{ }_{1} q_{0}}{1-\left(1-b_{0}\right) \cdot{ }_{1} q_{0}}=A \geq 0.107, \\
\frac{-\left[1-\left(1-c_{00}\right) \cdot{ }_{1} q_{0}\right]+\sqrt{\left[1-\left(1-c_{00}\right) \cdot{ }_{1} q_{0}\right]^{2}+4 \cdot c_{01} \cdot{ }_{1} q_{0}^{2}}}{2 \cdot c_{01} \cdot{ }_{1} q_{0}},
\end{array}\right. \\
& { }_{1} a_{0}=\frac{{ }_{1} q_{0} \cdot\left(1+{ }_{1} m_{0}\right)-{ }_{1} m_{0}}{{ }_{1} q_{0} \cdot{ }_{1} m_{0}}
\end{aligned}
$$

The values of ${ }_{4} a_{1}$ and ${ }_{4} m_{1}$ can be solved similarly:

$$
\begin{aligned}
& { }_{4} a_{1}=\left\{\begin{array}{c}
b_{1},{ }_{1} m_{0} \geq 0.107, \\
c_{10}+c_{11} \cdot{ }_{1} m_{0},{ }_{1} m_{0}<0.107,
\end{array}\right. \\
& { }_{4} m_{1}=\frac{{ }_{4} q_{1}}{4-\left(4-{ }_{4} a_{1}\right) \cdot{ }_{4} q_{1}},
\end{aligned}
$$

where the values of the parameters in (A.2)-(A.5) are in Table A.1.

Table A1. Parameters of (A.2)-(A.5).

\begin{tabular}{lrrrrrr} 
Male & .33 & 1.352 & .045 & 2.684 & 1.651 & -2.816 \\
Female & .35 & 1.351 & .053 & 2.8 & 1.522 & -1.518 \\
\hline
\end{tabular}

For other age groups, the values of ${ }_{n} a_{a}$ and ${ }_{n} m_{a}$ come from solving the equations below:

$$
\left\{\begin{array}{c}
{ }_{n} a_{a}=\frac{n}{2}-\frac{n^{2}}{12}\left[{ }_{n} m_{a}-\frac{{ }_{n} m_{a}-{ }_{n} m_{a-n}}{n \cdot{ }_{n} m_{a}}\right], \\
{ }_{n} m_{a}=\frac{{ }_{n} q_{a}}{n-\left(n-{ }_{n} a_{a}\right) \cdot{ }_{n} q_{a}},
\end{array}\right.
$$

of which the first is the Greville (1943) formula and the second is a definition. 
Having the values of $m_{a}$, the number of person-years lived between ages $a$ and $a+n$ is obtained as

$$
{ }_{n} L_{a}=\frac{{ }_{n} d_{a}}{{ }_{n} m_{a}}
$$

The other two life-table variables, namely, total person-years lived over age $a$ and life expectancy at age $a$, can then be computed as

$$
\begin{aligned}
& T_{a}=\sum_{y=a}{ }_{n} L_{y}, \\
& e_{a}=\frac{T_{a}}{l_{a}} .
\end{aligned}
$$

Finally, for the open age group starting at age $\omega,{ }_{\infty} \mathrm{L}_{\omega}=1 /{ }_{\infty} m_{\omega}(0)$.

The above formulas apply to abridged life tables. For complete life tables, of which the length of all age intervals is one year, (A.1)-(A.3) still apply for age group $[0,1),{ }_{\infty} \mathrm{L}_{\omega}=1 /{ }_{\infty} m_{\omega}(0)$ works also for the open age group, and (A.6)-(A.8) can be used for other age groups. 